\title{
Literacidad en el derecho. Aproximaciones a partir de un corpus de ensayos sobre argumentación jurídica ${ }^{1}$
}

\author{
Hugo Manuel Camarillo Hinojoza \\ Universidad Autónoma de Ciudad Juárez \\ Ciudad Juárez, México
}

\section{Resumen}

El derecho es lenguaje y la argumentación jurídica una actividad lingüística. Las habilidades y destrezas para la comprensión y producción textual son trascendentes para el aprendizaje y ejercicio profesional del derecho dado lo imprescindible y recurrente de la articulación del discurso oral y escrito. El objetivo del artículo es presentar los hallazgos relacionados con el dominio de literacidad funcional por parte de los estudiantes de Derecho de una universidad mexicana en el contexto de la literacidad académica. Utilizamos el análisis del discurso en un corpus de 36 ensayos sobre la importancia de la argumentación jurídica. Los resultados presentados corresponden a la estructura del texto, el manejo e inserción de voces y la construcción de perspectiva. Una de las conclusiones consiste en que los profesores deben motivar el desarrollo de destrezas en los estudiantes para la comprensión y producción textual para mejorar su capacidad argumentativa porque incidirá en su campo laboralprofesional.

Palabras clave: literacidad académica; literacidad funcional; literacidad disciplinar; literacidad jurídica; argumentación jurídica.

\footnotetext{
1 Este artículo se deriva de una investigación más amplia cuyo objetivo fue identificar aspectos o características de los tres enfoques de la literacidad: funcional, crítica y sociocultural en una unidad de análisis de 36 ensayos sobre la importancia de la argumentación jurídica en la vida profesional-laboral de los estudiantes de la licenciatura en Derecho de la Universidad Autónoma de Ciudad Juárez (UACJ) y que se presentaron como trabajo final en la asignatura de Argumentación Jurídica durante el semestre enerojunio de 2020. La investigación fue desarrollada sin el financiamiento de alguna institución y culminó el 30 de junio de 2020. El investigador principal es el autor del presente artículo.
} 


\section{Abstract \\ Literacy in law. Approaches from a corpus of essays on legal argumentation}

Law is language and legal argumentation is a linguistic activity. The skills and abilities for textual comprehension and production are essential for the learning and professional practice of law given the fundamental and recurrent nature of the articulation of oral and written discourse. The aim of the paper is to report the findings on functional literacy in 36 essays written by law students in a Mexican university in the context of academic literacy. The topic of the prompt essay was the importance of legal argumentation. Results in this paper are mainly concerned with the structure of the text, the handling and insertion of voices and stance-taking devices. One of the conclusions is that teachers must foster the development of text comprehension and production skills in law students to improve their argumentative competence because it will affect their professional practice.

Key words: academic literacy; functional literacy; disciplinary literacy; legal literacy; legal argumentation.

\section{Résumé}

La literacité dans le domaine du droit. Approches à partir d'un corpus d'essais sur l'argumentation juridique

136 Le droit est langage et l'argumentation juridique est une activité linguistique. Les habilités et les aptitudes pour la compréhension et production textuelle sont significatives pour l'apprentisage et l'exercice professionnel du droit vu l'importance et la récurrence de l'articulation du discour oral et écrit. L'objetif de cet article est de présenter des résultats concernant le domaine de la literacité fonctionnelle chez des étudiants en Droit d'une université mexicaine. Pour ce faire, l'on a analysé un corpus de 36 essais sur l'importance de l'argumentation juridique, à la lumiere de la théorie de l'analyse du discours. Les donnés correspondent à la structure du texte, la maîtrise et l'insertion de voix et la construction de perspective. Une des principales conclusions de cette étude est que les professeurs doivent motiver le développment des habilités pour la compréhension et production textuelle afin d'améliorer la capacité argumentative des étudiants. Celle-ci aura une incidence sur le domaine professionnel.

Mots-clés : literacité académique; literacité fonctionnelle; literacité disciplinaire; literacité juridique ; argumentation juridique. 


\section{SOBRE EL AUTOR}

\section{Hugo Manuel Camarillo Hinojoza}

Profesor-investigador de tiempo completo de la Universidad Autónoma de Ciudad Juárez (UACJ). Doctor en Ciencias Sociales, maestro en Educación y licenciado en Derecho. Miembro del Centro de Investigaciones Jurídicas y Coordinador de la Academia de Teoría General del Derecho de la UACJ. Sus áreas de docencia son: la metodología de la investigación jurídica y la argumentación jurídica. Sus áreas de investigación son: el aprendizaje del derecho y la evaluación de los académicos.

Correo electrónico: hcamaril@uacj.mx

\section{CóMO CITAR ESTE ARTÍCULO}

Camarillo, H. (2021). Literacidad en el derecho. Aproximaciones a partir de un corpus de ensayos sobre argumentación jurídica. Lenguaje, 49(1), 135-164. https://doi.org/10.25100/lenguaje.v49i1.10485. 


\section{INTRODUCCIÓN}

Para la elaboración del presente artículo partimos de la aseveración que expresó Aarnio (2008) respecto a que el derecho es lenguaje. Asimismo coincidimos con Cáceres (2000) en que el derecho está lleno de proposiciones lingüísticas. Por tanto, cuando en el contexto del derecho se estructuran argumentos se suscita una actividad que no deja de ser lingüística (Atienza, 2006, 2013; Cárdenas, 2018).

El ejercicio y la práctica del derecho requieren de la elaboración de discursos escritos y orales coherentes. Esto se puede identificar en los distintos géneros discursivos jurídicos. Por ejemplo, un juez al aplicar el derecho a un caso concreto a través de la emisión de una sentencia debe construir argumentos sólidos que sustentan su decisión judicial.

En este sentido, las habilidades y destrezas para la comprensión y producción textual son trascendentes para el aprendizaje del derecho y su ejercicio profesional en virtud de que resulta imprescindible la articulación de discursos. En esto radica la importancia de desarrollarlas y promoverlas durante la formación académica de los estudiantes de derecho, que en el futuro pueden convertirse en jueces, legisladores, abogados litigantes, entre otros operadores jurídicos.

Bajo este contexto, en el artículo presentamos los hallazgos relacionados con el dominio de la literacidad funcional en los estudiantes de derecho de la UACJ a partir del análisis del discurso expresado en un género discursivo: el ensayo. El corpus analizado está comprendido por 36 ensayos jurídicos que fueron presentados como trabajo final en la asignatura de Argumentación Jurídica durante el semestre enero-junio de 2020 por parte de estudiantes de pregrado en derecho de la UACJ. El análisis de este corpus se hizo con base en la propuesta de Castro y Sánchez (2013) que referimos en el apartado metodológico.

El artículo se divide en cuatro partes. La primera trata sobre la literacidad y sus enfoques, la vigencia de la literacidad funcional, la importancia de la comprensión y producción textual en el aprendizaje y ejercicio profesional del derecho, y sobre la argumentación jurídica como actividad lingüística. También hacemos una revisión de los trabajos más recientes sobre literacidad jurídica, ya que este trabajo se ubica en el contexto de una literacidad disciplinar. En la segunda parte describimos la metodología utilizada para la aproximación epistemológica y el análisis de los datos realizado a través del análisis del discurso. La tercera corresponde a los hallazgos encontrados que se presentan en tres secciones consistentes en: a) la estructura del texto; b) la inserción y manejo de voces y c) la construcción de perspectiva. En la última parte se presentan algunas de las reflexiones finales sobre el dominio de la literacidad en los estudiantes de derecho de la universidad pública mencionada. 


\section{Literacidad. Un concepto complejo}

En ocasiones ocurre que la literacidad como concepto se relaciona con el de alfabetización. Sin embargo, como señala Zavala (2004), desde luego son vocablos distintos e implican significados particularmente especiales. Siguiendo a Cassany y Castellà (2010), lo anterior se deriva de las confusiones producidas por la traducción de literacy. Incluso, de manera reciente a la aparición del término literacidad en América Latina, algunos autores como Kalman (1993) advertían de la complejidad de lo que en ese momento se configuraba como una nueva palabra.

Definir la literacidad no resulta una tarea sencilla. Desde nuestra perspectiva, responder a ¿qué es la literacidad? configura un serio y exhaustivo problema por dos razones fundamentales: en primer lugar comprende tres enfoques: a) funcional; b) crítico y c) sociocultural; y en segundo lugar, cada uno de estos se deriva de determinados posicionamientos teóricos y epistemológicos, lo que de alguna manera hace que se potencialice su complejidad.

\section{El enfoque funcional de literacidad y su vigencia teórico-pragmática}

En principio, pareciera que este enfoque es menos complejo que los otros dos. Como señalan Riquelme y Quintero (2017), el enfoque funcional estuvo vigente en la discusión hasta la década de los ochenta. Luego los enfoques de carácter crítico y sociocultural comenzaron a cobrar fuerza teórica y empírica al considerarse que la literacidad comprendía "habilidades más amplias que la decodificación y comprensión de lo escrito" (Márquez y Valenzuela, 2018, p. 2). De esta manera, el análisis de la literacidad permitió darle un sentido más crítico. Fue posible aseverar que transforma las vidas de las personas porque las emancipa, las libera, las empodera a través del discurso (Cassany, 2005). Además, los Nuevos Estudios de Literacidad (NEL) argumentaron con sustento que la literacidad era una práctica ideológica (Street, 2004) o, como refiere Gee (2004), una práctica discursiva vinculada a cómo se percibe el mundo y la realidad social por parte de los grupos sociales.

En todo lo anterior coincidimos con los autores citados; sin embargo es pertinente precisar que el enfoque funcional también resulta complejo. Sobre todo porque comprende cuatro dimensiones que proponemos: 1) habilitadora; 2) dinámicoparticipante; 3) informacional y 4) digital-tecnológica en razón de determinados aspectos que resaltan a partir de su análisis. A esta conclusión llegamos interpretando a algunos autores. Por ejemplo, importa el desarrollo de habilidades, destrezas y capacidades de la comprensión y producción textual (Tejada y Vargas, 2007). La comprensión lectora es relevante, es decir, trasciende ser capaz de interpretar y comprender los códigos del lenguaje escrito y representarlos con otros. Esta sería la 
primera dimensión, que denominamos habilitadora, que se va configurando de forma pasiva.

En cambio la segunda es activa porque se requiere ponerla en movimiento. De acuerdo con Riquelme y Quintero (2017) y Braslavsky (2003), no basta el desarrollo de habilidades y capacidades, sino que es pertinente la participación social y laboral de la persona. Esto implica su desenvolvimiento satisfactorio ante las exigencias del entorno. Por esto la llamamos dimensión dinámico-participante.

Pero Larraín (2002) advirtió que no era suficiente un desarrollo de habilidades de lecto-escritura para ser funcional socialmente. Expuso sobre la capacidad en el manejo y proceso del cúmulo de información que había invadido el mundo. Esta sería la tercera dimensión, que denominamos informacional.

Sin embargo, debido a la información que se produce cada vez más, que se maneja y comparte por los seres humanos a través de las redes sociales y plataformas electrónicas en las que también se utilizan códigos lingüísticos, incluso distintos de los tradicionales porque actualmente "vivimos colgados y dependientes de lo digital" (García Aretio, 2019, p. 11), se requiere del desarrollo y manejo de habilidades digitales. Por lo que se trata entonces de la relación de la literacidad con las Tecnologías de Información y Comunicación (TIC) (Braslavsky, 2003; Guerrero, 2019), de los planteamientos de cómo ha impactado la cultura digital a la literacidad (Cassany, 2006), de las prácticas actuales entre la literacidad y la escuela (Tapia, 2019) en razón del grado de posicionamiento de la lectura a través de medios electrónicos (Salado et al., 2017; Salado y Ramírez-Matinell, 2018) y cómo todo lo anterior influye actualmente sobre los procesos de comprensión textual (Márquez y Valenzuela, 2018) y aprendizaje en ciertas disciplinas (Camarillo y Barboza, 2020). Es decir que lo anterior comprende lo que se considera como literacidad digital, por lo que, bajo este panorama, finalmente se configuraría la cuarta dimensión de la literacidad funcional, que denominamos digitaltecnológica.

A partir de lo anterior y como se pudo advertir, consideramos que las cuatro dimensiones citadas le dan vida al enfoque funcional de la literacidad. Por esta razón se apologiza su complejidad, su vigencia teórico-pragmática y se propone lo que sería un modelo o representación tetradimensional de la literacidad funcional. Sin embargo, para los efectos particulares de este artículo y de acuerdo con el objetivo propuesto, haremos referencia solo a la primera de las dimensiones: la habilitadora. Esto porque tiene relación con la pertinencia del desarrollo de habilidades y destrezas para la lectoescritura y la comprensión de textos en el caso de los estudiantes de Derecho durante su proceso de aprendizaje. 


\section{La literacidad académica y la literacidad disciplinar en el ámbito jurídico}

De acuerdo con lo afirmado por Lea y Street (2006), el contexto de la literacidad académica se encuentra en las áreas disciplinarias o en cursos temáticos, discursos institucionales, así como en la aproximación al conocimiento disciplinario por parte de los estudiantes. Para estos autores, en la literacidad académica existen tres modelos que pueden coexistir: a) modelo de habilidades de estudio; b) modelo de socialización académica y c) modelo de literacidades académicas. Además refieren que la literacidad académica implica analizar la escritura y las prácticas de literacidad en la biología, en la antropología - por dar ejemplos- o en alguna otra área de la ciencia.

Desde nuestra perspectiva, esto es de considerarse por las instituciones de educación superior y el profesorado en razón de problemáticas que sabemos existen en el proceso de enseñanza-aprendizaje, y que Lea y Street (2006) advierten claramente. Argumentan que uno de los problemas a los que se enfrentan los estudiantes que ingresan a la universidad está relacionados con la escritura y el discurso académico, por lo que esta debilidad debe atenderse para su fortalecimiento.

En el señalamiento de los autores mencionados se identifica la importancia y trascendencia de la literacidad académica. A su vez, para Carlino (2013) implica el:

acceso de los estudiantes a las diferentes culturas escritas de las disciplinas. Es el intento denodado por incluirlos en sus prácticas letradas, las acciones que han de realizar los profesores, con apoyo institucional, para que los universitarios aprendan a exponer, argumentar, resumir, buscar información, jerarquizarla, ponerla en relación, valorar razonamientos, debatir, etcétera, según los modos típicos de hacerlo en cada materia. Conlleva dos objetivos que, si bien relacionados, conviene distinguir: enseñar a participar en los géneros propios de un campo del saber y enseñar las prácticas de estudio adecuadas para aprender en él. En el primer caso, se trata de formar para escribir y leer como lo hacen los especialistas; en el segundo, de enseñar a leer y a escribir para apropiarse del conocimiento producido por ellos. (p. 370).

A partir de los planteamientos de Lea y Street (2006) y Carlino (2013), se identifica lo medular de la literacidad académica en las disciplinas. Por lo que es pertinente referir lo que comprende la literacidad disciplinar, "cuya premisa consiste en que el conocimiento de las disciplinas es siempre discursivo y mediado por textos" (Moreno, 2019, p. 96). Conforme a Montes y López (2017) es:

un tipo de práctica de literacidad que se da en el marco de un dominio específico y restringido; una disciplina donde la lectura y la escritura se ven como prácticas inherentes y esenciales, las cuales pueden ser reproducidas, aprendidas y enseñadas. (p. 165). 
De acuerdo con ambos trabajos, la literacidad disciplinaria se trata de prácticas de literacidad circunscritas a una área o cultura disciplinar específica en la que la comprensión y producción textual se erigen como esenciales. Así, cada disciplina tiene sus propias dinámicas o prácticas letradas que realizan los especialistas en las que se incorporan los estudiantes a través de actividades. "Es decir, la formación del novel profesionista consistiría en aprender un lenguaje y, a la vez, apropiarse de un acto profesional total o forma de pensar el mundo, de sentir y de actuar en él de manera profesional" (Farfán, 2019, p. 10).

Conforme a los planteamientos de Lea y Street (2006), Carlino (2013), Moreno (2019), Montes y López (2017), Farfán (2019), entre otros autores, los estudios de literacidad focalizados en las disciplinas son pertinentes por las razones que ellos mismos expresan. El presente trabajo se inserta em los estudios de literacidad disciplinar porque se aborda la temática en el ámbito jurídico.

La justificación emerge por sí sola dado que "el derecho, al igual que muchas otras disciplinas, cuenta con un lenguaje técnico especializado, con diferentes grados de generalidad" (Cáceres, 2000, p. 60) que los estudiantes deben dominar durante su formación académica y durante el ejercicio profesional. Los estudiantes aprenden derecho leyendo teoría, leyes, jurisprudencias, entre otros documentos jurídicos (Carbonell, 2014). Además, una vez que egresan los estudiantes de la licenciatura y comienzan a ejercer profesionalmente, la práctica de la comprensión y producción textual sigue constituyendo una prioridad a la hora de hacer, por ejemplo, la interpretación de las normas jurídicas (Carbonell, 2014).

Conforme a Cáceres (2000), el derecho "significa un sistema de normas de cierta índole, las cuales son una clase de proposiciones lingüísticas" (p. 11). Como precisa Aguirre (2008), la relación entre derecho y el lenguaje se puede resumir en dos enfoques: uno denominado instrumentalista, porque el derecho usa al lenguaje para llegar a sus destinatarios, y otro considerado constitutivo, sustentado en que el derecho es lenguaje. Esta última afirmación ya la había expresado Aarnio (2008). Para este autor el derecho es lenguaje porque las normas jurídicas, las sentencias judiciales, la técnica legislativa y la interpretación del derecho se materializan en lenguaje, y la estructuración del discurso jurídico debe contener un lenguaje argumentado. Para Atienza (2006, 2013), cuando se argumenta jurídicamente estamos hablando de una actividad lingüística.

Si bien las otras disciplinas también se expresan mediante lenguaje, en el caso del derecho el lenguaje escrito y el oral configuran una esencialidad porque, por ejemplo, las normas jurídicas se encuentran sistematizadas en codificaciones escritas que se interpretan bajo métodos para aplicarse en los tribunales para resolver los litigios conforme a lo que obra en expedientes jurídicos. De tal manera que los casos planteados ante el juez deben redactarse en escritos de demandas lo suficientemente argumentados jurídicamente -o como dirían Baquero y Pardo (1997, 1998)- con una estructura argumentativa. A su vez, dentro de los juicios se desarrollan audiencias públicas en las 
que los abogados hacen uso de su retórica jurídica para persuadir y convencer, preguntar y repreguntar, externar alegatos, objetar, impugnar, entre otras acciones. Así pues, en el contexto de la literacidad jurídica -por citar un ejemplo solo en cuanto al lenguaje escrito- los estudiantes de derecho deben, por un lado: a) prepararse para desarrollar habilidades, destrezas y capacidades para interpretar los textos de las normas jurídicas creadas por los legisladores, analizar y comprender discursos plasmados en jurisprudencias, sentencias, expedientes jurídicos, libros, artículos de revistas y escritos jurídicos de diferentes ramas del derecho que utilizan los especialistas de la disciplina; y por el otro lado: b) prepararse para desarrollar habilidades, destrezas y capacidades para redactar de manera organizada, argumentada y fundamentada en diferentes discursos jurídicos dado que los estudiantes de derecho tienen como campo de acción en su ejercicio profesional una amalgama de posibilidades en el contexto de lo legislativo, judicial, ejecutivo, notarial, del litigio, la docencia, la investigación, entre otros.

Bajo este contexto, la literacidad académica y la literacidad disciplinar son sumamente relevantes para el derecho. Sobre todo en lo referente a la dimensión habilitadora de la literacidad funcional que mencionamos en el apartado denominado "El enfoque funcional de literacidad y su vigencia teórico-pragmática", de este artículo. ¿Por qué? La razón es que durante el proceso de aprendizaje de los estudiantes de derecho es imprescindible desarrollar el conjunto de habilidades, destrezas y competencias que expusimos en el párrafo anterior. Desde luego Lea y Street (2006) tienen razón cuando afirman que la literacidad académica percibe a la escritura y al aprendizaje de los estudiantes más como un aspecto epistemológico que una cuestión de desarrollar o propiciar habilidades en los estudiantes; sin embargo, en el caso específico de los estudiantes de derecho, la "habilitación" se configura como imprescindible, ya que sin las "habilidades, destrezas y capacidades" planteadas anteriormente y que están relacionadas con una literacidad académica y disciplinar; entonces los estudiantes de derecho no serían funcionales en su práctica profesional jurídica.

Desde luego el desarrollo de prácticas de literacidad disciplinar no es exclusivo para los estudiantes o egresados de derecho, sino también para aquellos que se caracterizan por contar con experiencia y práctica profesional. De acuerdo con Taranilla (2012) "la práctica del derecho es una actividad eminentemente lingüística" (p. 1), por lo que, en cualquiera de las vertientes del ejercicio profesional del derecho -el litigio, la función judicial, la función legislativa, la academia y más- la literacidad académica y disciplinar debe estar presente. Un ejemplo de lo anterior está documentado en el trabajo de González de la Torre (2011), quien analiza las prácticas de lectura en una escuela de derecho y en un juzgado civil. La autora concluye que: 
la escuela, a través de la actividad que se produce de manera predominante, dispone a los sujetos a una práctica lectora en la cual el motivo central es la presentación e intercambio de información, en un escenario donde se pretende de manera implícita dotar a los estudiantes de una imagen profesional, y por consecuencia se insiste en que se apropien de elementos de la cultura de la profesión, como agregado a los conocimientos que circulan en el aula. Sin embargo, el sujeto que ha leído en un contexto escolar, al incorporarse a un contexto diferente en el que cambia su rol, responsabilidades y objetivos de lectura, tiene que reorientar su práctica lectora. Se puede concluir, a partir de los datos analizados, que en el trabajo, la posibilidad de una lectura eficaz se basa principalmente en el conocimiento previo del trabajador, conocimiento adquirido en gran parte como resultado de su trayectoria escolar. (González de la Torre, 2011, p. 49).

En suma, el desarrollo de habilidades y competencias para leer y escribir, así como elevarlas a un nivel de reflexión y comprensión de textos son pertinentes en el campo jurídico. Es aquí donde la literacidad funcional cobra relevancia y sentido para la profesión. En esto se sitúa la justificación del presente artículo y su aporte a los estudios sobre literacidad jurídica.

\section{La literacidad en el campo especializado del derecho}

En América Latina los estudios acerca de la literacidad jurídica no han sido prolíficos (Londoño et al., 2019). Este desarrollo de alguna manera incipiente es absurdo pues como aseveramos anteriormente, la comprensión y producción textual son medulares para el aprendizaje del derecho y su ejercicio profesional. Por tanto deben realizarse más estudios.

Un trabajo publicado hace una década refiere la intrínseca relación entre la literacidad y el derecho (Moreno Mosquera, 2010). Afirma que los profesionales del derecho deben tener desarrolladas destrezas y habilidades para la comprensión y producción textual, así como para la interpretación y comprensión de la normativa. Retoma aspectos de un debate entre el sentido formalista del lenguaje en el derecho y el antiformalista.

Por su parte González de la Torre (2011) documenta bajo una metodología etnográfica cómo se suscitan prácticas de lectura en estudiantes de derecho y otra disciplina de una universidad pública mexicana. Además, presenta resultados sobre cómo se realiza la práctica lectora de actores que conforman el personal de un juzgado civil y de otra institución pública.

En otro de sus trabajos, Moreno Mosquera (2012) resaltó la importancia de la argumentación jurídica en los estudiantes de derecho de una universidad colombiana 
desde un enfoque crítico de la literacidad. Refiere la trascendencia de los razonamientos en la disciplina jurídica. Sin embargo el autor se posiciona en favor de una argumentación crítica referida como pragmadialéctica en lugar de una argumentación solamente retórica porque sostiene que así se pondera el contexto histórico-político. El autor propone como necesario buscar:

nuevas alternativas argumentativas, como la teoría de los actos de habla, fruto de una propuesta de la Pragmática y la Filosofía del Lenguaje desde el Derecho, con el ánimo de romper el paradigma tradicional y crear una visión crítica del estudiante en su proceso de aprendizaje, que inexorablemente lo conducirá a la investigación. (Moreno Mosquera, 2012, p. 126).

Por su parte Taranilla (2012) menciona algunos de los trabajos más sobresalientes en inglés sobre la lingüística en el derecho y las destrezas comunicativas en lo referente al proceso judicial. Afirma que "en el quehacer de los profesionales del derecho desempeña un papel crucial su competencia comunicativa" (p. 1). Refiere que la palabra es el centro de la construcción y transmisión del conocimiento jurídico. El artículo se focaliza en las competencias narrativas en el ejercicio del derecho penal, por lo que constituye un aporte al enfoque funcional de la literacidad. En este sentido analiza las narrativas de cinco géneros discursivos que están presentes en el proceso penal español. La autora profundiza en los siguientes: a) el escrito de acusación, b) el escrito de defensa, c) el interrogatorio y la toma de declaración pericial, d) informe final y e) sentencia.

El trabajo de García Petit y Agüero (2014) se sitúa en un enfoque sociocultural. Considera que el discurso jurídico es una práctica social porque mediante este se vincula la comunidad experta en el conocimiento jurídico con la sociedad. Argumenta que los aspectos sociales, políticos y culturales impactan en el discurso ideológico de un sistema jurídico. Para estos autores el "lenguaje es una institución política y dominarlo exige tener conciencia explícita de qué recursos lingüísticos están socialmente asociados a diferentes contextos de interacción y campos de conocimiento" (García Petit y Agüero, 2014, pp. 64-65). En este sentido, la comunidad jurídica domina el lenguaje exclusivo de la profesión pero, a su vez, no se encuentra aislado de lo social.

En el trabajo de Betancourt y Frías (2015) se desprende una coincidencia con el de Moreno Mosquera (2012) en cuanto a la pertinencia del desarrollo de las competencias argumentativas. Pero mientras este autor se posiciona en una argumentación jurídica desde el enfoque crítico de la literacidad, Betancourt y Frías (2015) la ponderan desde el enfoque funcional. Afirman que las habilidades y destrezas argumentativas son imprescindibles para un desempeño eficaz de los profesionales del derecho en el mundo laboral. Advierten de la urgencia de desarrollar habilidades de lecto-escritura y de interpretación lingüística durante la formación académica de los estudiantes de derecho, dadas las debilidades que encontraron en su estudio. "En este 
sentir, la argumentación y la literacidad terminan representadas en los límites enmarcados del lenguaje, posibilitándose en la medida de apropiación de los códigos de estructuración lingüística que se utilicen en la discusión argumentativa" (Betancourt y Frías, 2015, p. 217) ya que la "argumentación es uno de los pilares de la formación jurídica que sustenta el perfil profesional de los abogados" (p. 218).

También Henao et al. (2017) señalaron en su trabajo problemas de literacidad en los estudiantes de derecho. Documentaron sus experiencias con la argumentación pragmadialéctica, la lectura crítica y la textualidad en el contexto de la asignatura de Técnicas Comunicativas del programa educativo de derecho de la Institución Universitaria de Envigado de Colombia. Concluyeron que si bien los estudiantes presentaban deficiencias de literacidad al inicio del curso hubo un avance significativo en cuanto a la textualidad. Además, subrayaron que para poder hacer un buen uso de la argumentación es medular el desarrollo de habilidades en lo referente a la lectura crítica, así como en la construcción de resúmenes en razón de un proceso que condensa e integra la información contenida en el discurso.

En otros trabajos, como el de Aguilar (2017), se pone atención a los distintos géneros discursivos jurídicos. La autora presentó un análisis de las funciones que cumplen particularmente tres grupos: "los relativos a la escritura como destreza profesional, aquellos de investigación jurídica del estudiante y los que tienen por propósito la creación de conocimiento jurídico" (p. 179). La clasificación es amplia y exhaustiva dado que son recurrentes estos géneros durante la enseñanza-aprendizaje del derecho. Para Aguilar (2017):

En lo que respecta a la actividad jurídica, ésta amerita una especial atención en la enseñanza formal de ciertas estrategias de análisis, mecanismos de argumentación y persuasión; en definitiva, requiere el desarrollo de habilidades comunicativas escritas y orales debido a la importancia que tiene la precisión léxica y retórica en la fundamentación y explicación de ideas y conceptos en diversos contextos comunicativos. (p. 183).

Finalmente, en un trabajo reciente, Londoño et al. (2019) estudiaron los niveles de literacidad en las prácticas de los docentes de las facultades de derecho de una Institución de Educación Superior (IES) privada y otra pública de Colombia. Se trata de una investigación cualitativa en la que preguntaron a los docentes de derecho cómo conciben la comprensión y producción textual en su área disciplinaria. Documentaron que la totalidad de sus entrevistados afirmaron sobre la importancia de la lectura y la escritura en la formación académica para la abogacía. Presentan los resultados sistemáticamente conforme a las siete categorías de análisis: géneros discursivos, campo jurídico, argumentación jurídica, intertextualidad, didáctica, prácticas textuales y oralidad. Concluyen que es pertinente que los docentes de derecho tengan un 
acompañamiento para que puedan acentuar prácticas pedagógicas y lingüísticas en las que se fortalezcan la comprensión de textos y su producción en los estudiantes.

\section{Metodología}

Este trabajo se desprende de un segundo análisis en el marco de una investigación más amplia en la que utilizamos una metodología cualitativa, de corte interpretativo, posicionada en el análisis del discurso (Du Bois, 2003, 2007, 2014), en virtud de que el estudio se focaliza en una unidad de análisis consistente en 36 ensayos jurídicos sobre la importancia de la argumentación jurídica en la vida laboral-profesional, ensayos que fueron redactados por estudiantes de derecho de nivel intermedio como trabajo final de la asignatura de Argumentación Jurídica, grupo A, de la licenciatura en Derecho de la UACJ durante el semestre enero-junio de 2020.

Coincidimos con Calsamiglia y Tusón (2012) en que el análisis del discurso constituye un instrumento para la comprensión de las prácticas discursivas que emergen en cualquier entorno o contexto en los que la palabra oral y escrita es medular; $\mathrm{y}$, por ejemplo, ya argumentamos en los apartados anteriores que esto ocurre en el aprendizaje y ejercicio del derecho debido a que la disciplina está íntimamente relacionada con el lenguaje. De tal manera que en los ensayos que conformen la unidad de análisis los estudiantes se vincularon a través de sus discursos con la esfera social profesional-laboral debido a la temática del texto. Esta vinculación permite identificar, mediante el análisis del discurso, su nivel de literacidad funcional en el contexto de la literacidad en el ámbito jurídico. Como sostiene Aguilar (2017), "una de las aplicaciones prácticas del análisis del discurso es el estudio de instancias comunicativas auténticas y la descripción de las relaciones entre el lenguaje y sus posibles contextos comunicativos de producción" (p. 184); el ámbito profesional-laboral jurídico funge como ese contexto de acuerdo con el tema principal del ensayo redactado por los estudiantes.

Además, coincidimos con González Lillo (2020) en que escribir "es una de las habilidades más necesarias que desarrollar en la vida de todo sujeto, (...). Por lo tanto, la escuela ha de tener el propósito de formar niños y jóvenes competentes en producción escrita" (p. 331). De tal forma que analizar los ensayos de los estudiantes de derecho como textos comunicativos y dialógicos es pertinente dado que las instituciones de educación superior tienen un compromiso con la literacidad académica (Aguilar, 2017).

Algunos de las interrogantes que pueden emerger son: ¿Por qué elegir el ensayo como género discursivo para realizar el análisis, en lugar de otros aparentemente más acordes con el campo del derecho, como sería el caso de un escrito de acusación, una demanda, entre otros, por sus características netamente jurídicas? y ¿Por qué elegir una unidad de análisis consistente en 36 ensayos en los que estudiantes de derecho se expresaron sobre la importancia de la argumentación en su vida profesional-laboral, para encontrar aspectos o características de su dominio de literacidad funcional en el 
contexto de la literacidad académica y disciplinaria en el ámbito jurídico? Entre las respuestas se encuentran que el ensayo representa un posicionamiento discursivo y constituye un texto en el que puede haber una interacción dialógica entre lector y autor (Castro y Sánchez, 2013), en el que este manifiesta de forma libre sus reflexiones y perspectivas sobre un tema. Además, en otros trabajos (Castro et al., 2010; Parodi, 2010) se analiza el ensayo como género discursivo en las prácticas de escritura en el nivel universitario.

Además, en el estudio que realizamos, no se trata solo de un ensayo en general, sino de uno de carácter jurídico relacionado con la argumentación jurídica, cuya trascendencia para cualquier rama del derecho es medular. La argumentación jurídica es una disciplina y una actividad imprescindible para el estudio y la práctica del derecho. Sin ella el derecho no se puede ejercitar porque implica la exposición de razones sostenibles y fundamentadas. Hay que "entender la argumentación como derecho, o viceversa" (Cárdenas, 2018, p. 8) y la argumentación como una actividad lingüística (Atienza, 2006, 2013). Como precisa Cárdenas (2018), en "las teorías de la argumentación importa cómo se argumenta, cómo se motiva, cómo se descubren los argumentos, cuáles son sus tipos y cómo se puede evitar la manipulación de los instrumentos retóricos" (p. 24).

Por tanto, un ensayo en el que los estudiantes de derecho expongan sus ideas respecto a lo que ellos piensan o consideran en torno a la importancia de la argumentación jurídica en su vida profesional-laboral, resulta acorde metodológicamente hablando para analizar su nivel de dominio de literacidad funcional en el rubro de la habilitación, mencionado en el apartado denominado "El enfoque funcional de literacidad y su vigencia teórica-pragmática", en el contexto de la literacidad académica y disciplinar, ya que la argumentación jurídica se utiliza en cada una de las diferentes ramas del derecho: civil, familiar, mercantil, penal, laboral, constitucional, amparo, fiscal, etc. En este sentido, como afirman Betancourt y Frías (2015), "la argumentación y la literacidad terminan representadas en los límites enmarcados del lenguaje, posibilitándose en la medida de apropiación de los códigos de estructuración lingüística que se utilicen en la discusión argumentativa" (p. 217).

Asimismo, si bien en trabajos como el de Taranilla (2012) y Aguilar (2017) se refieren como géneros discursivos del derecho al escrito de defensa, al interrogatorio, a la declaración pericial, a las demandas, a los escritos de apelación, entre otros, esto no significa que el ensayo no pueda constituir un género aplicado al derecho. Por ejemplo, el mismo artículo de Aguilar (2017) sí lo considera como un género discursivo del derecho, aunque lo clasifica dentro de la generalidad en los relativos a la investigación jurídica y conforme a su particularidad lo ubica como un texto de nivel inicial, orientado a la exposición de problemáticas y con una función discursiva que lo familiariza con la escritura académica. Sin embargo, en contraste parcialmente con Aguilar (2017), nosotros situamos al ensayo jurídico como un género discursivo en el que es posible 
identificar, además de hallazgos sobre el dominio de aspectos para la investigación jurídica, hallazgos sobre el dominio de una literacidad funcional en el contexto de la literacidad académica.

En este sentido, el artículo se enmarca en un trabajo de literacidad disciplinar al estudiarse aspectos relacionados con la literacidad jurídica a través del análisis de un género discursivo particular elaborado por estudiantes de derecho cuyo tema fue el impacto e importancia de la argumentación jurídica en su vida laboral-profesional.

Con la intención de que la totalidad de los ensayos tuviera ciertos ejes que organizaran mejor el análisis, decidimos plantear a los estudiantes algunos aspectos para que fueran ponderados por ellos durante la redacción de sus trabajos. Atendiendo a las recomendaciones de Parodi (2010), a continuación se describen los ensayos que se leyeron y analizaron:

1.- Los 36 ensayos configuraron el trabajo final por presentar en la asignatura de Argumentación Jurídica. Como trabajo de fin de semestre se esperaba que los estudiantes hicieran mención de temas y aspectos vistos en clase, ideas de los autores revisados, dinámicas pedagógicas realizadas en el curso, ejercicios prácticos de argumentación, etc., para analizar a través de sus discursos escritos su dominio de literacidad funcional en cuanto a su vida profesional-laboral a partir de la importancia de la argumentación jurídica en esta esfera social.

2.- El tema general del ensayo radicó en el impacto de la Argumentación Jurídica en su vida profesional-laboral en cualquiera de sus vertientes: ejercicio de la abogacía, la función jurisdiccional, la actividad legislativa, la academia, entre otras. Se les hizo saber que podían elegir una o varias. Esto porque no todos tenían una práctica jurídica fuera de la escuela. De tal manera que se dejó abierta la proyección que quisieran hacer a través de su discurso vinculado con la vida profesional-laboral jurídica. Y que se supone que dominar la argumentación jurídica los hace tener habilidades, destrezas y competencias en su disciplina. Aquí es donde se resalta la literacidad funcional, específicamente en cuanto a la dimensión habilitadora del modelo presentado en el apartado: "El enfoque funcional de literacidad y su vigencia teórico-pragmática" de este artículo.

3.- El contenido del ensayo se sustentó en al menos uno de los siguientes rubros: a) las lecturas (libros, capítulos de libros, artículos de revistas, etc.) correspondientes a los autores vistos en las sesiones de clase, b) las anotaciones o reflexiones que cada estudiante hizo a partir de la exposición del profesor en las clases y c) las dinámicas pedagógicas consistentes en diferentes ejercicios argumentativos realizados en las clases.

4.- El ensayo tuvo la siguiente estructura: introducción, desarrollo y conclusión con la finalidad de guardar cierta uniformidad en el corpus de 36 trabajos finales y para que los estudiantes pudieran exponer su opinión y reflexionar sobre el tema de una manera más organizada. 
5.- Finalmente, en cuanto a la descripción del ensayo, tuvo una extensión de entre 5 y 10 cuartillas. Además se redactó en primera persona en virtud del tema.

Es preciso destacar dos cuestiones relacionadas con la aproximación epistemológica y al análisis de los datos: la primera es que este trabajo se desprende de una investigación más amplia cuyo objetivo consistió en identificar aspectos o características de los enfoques funcional, crítico y socio cultural de la literacidad en la unidad de análisis, por lo que para el primer análisis y de acuerdo con su objetivo de investigación, atendimos el proceso de análisis propuesto por Castro y Sánchez (2013) con algunas modificaciones y ponderando ciertos planteamientos de Hernández (2017). La segunda cuestión se relaciona con que el presente trabajo se focalizó en el dominio de literacidad funcional (exclusivamente). Por tanto fue necesario hacer un segundo análisis -en el marco de la investigación más amplia- por lo que recurrimos de manera íntegra y sin modificaciones a los tres aspectos propuestos por Castro y Sánchez (2013) para el análisis de ensayos en los que se expresa la opinión de los estudiantes: a) estructura del texto, b) inserción y manejo de voces y c) construcción de perspectiva. Esto nos permitió identificar rasgos y características de una de las cuatro dimensiones de la literacidad funcional: la habilitadora; y posteriormente la comprensión del nivel de literacidad de los estudiantes de derecho que redactaron los ensayos que comprenden la unidad de análisis. Los ensayos fueron analizados uno a uno, apartado por apartado, y luego se integraron los resultados siguiendo los tres aspectos que proponen los autores mencionados para una comprensión general del corpus.

\section{RESULTADOS Y DISCUSIÓN}

\section{La literacidad y la capacidad para la argumentación jurídica}

En este apartado se presentan los hallazgos respecto al nivel de literacidad funcional encontrados durante el análisis del discurso de los ensayos presentados por los estudiantes de derecho de la UACJ en el curso de Argumentación Jurídica durante el semestre enero-junio de 2020.

Como refiere Moreno Mosquera (2010), es preponderante el desarrollo de habilidades y destrezas de lecto-escritura en los profesionales jurídicos. Incluso debiera estar presente como eje central en los diseños curriculares de los programas educativos de derecho. Esta habilitación es trascendente porque a final de cuentas -siguiendo a Aarnio (2008)- el ejercicio profesional del derecho en cualquiera de sus vertientes se materializa a través del uso del lenguaje. Además porque, con sustento en el trabajo de Valerdi (2020), consideramos que en el campo del derecho se suscita una influencia retórica del lenguaje en los discursos tanto orales como escritos.

Por tanto, identificar niveles de literacidad funcional en los ensayos cuya temática fue el impacto e importancia de la argumentación jurídica en la vida laboral- 
profesional de los estudiantes de derecho resulta pertinente dado que, como ya hemos multicitado, los autores consideran que el derecho es argumentación (Atienza, 2006, 2013; Cárdenas, 2018). Simplemente, por un lado "no puede concebirse el derecho contemporáneo si prescindimos de la argumentación" (Cárdenas, 2018, p. 8) y por el otro, la argumentación es una actividad lingüística (Atienza, 2006, 2013). Además, la literatura especializada en literacidad jurídica sostiene como fundamental el desarrollo de las capacidades argumentativas como parte de la esencia del profesional jurídico (Betancourt y Frías, 2015; Henao et al., 2017; Moreno Mosquera, 2012).

\section{El dominio de la literacidad funcional en los estudiantes de derecho de la UACJ}

En este sentido, al analizar el discurso de los 36 ensayos elaborados como trabajo final para la asignatura mencionada coincidimos con Castro y Sánchez (2013) en que:

En educación no se puede hablar de desarrollo de competencias argumentativas de modo independiente de los procesos de enseñanza y de aprendizaje. Nuestros estudiantes necesitan desarrollar habilidades que les permitan relacionar su capacidad de seleccionar y discriminar aquellos datos que les serán útiles para integrar los repertorios de conocimientos que exige el dominio de los contenidos de los cursos disciplinares. El dominio de las aptitudes persuasivas es central para que se incorporen de manera propositiva en las disquisiciones que se generan en la dinámica del desarrollo epistemológico de las diferentes áreas el conocimiento científico y humanístico. Estamos convencidos de que el sujeto que desarrolla sus habilidades retóricas y discursivas en el medio académico está mejor pertrechado para alcanzar notoriedad y éxito profesional, pero también se forja como ciudadano más consciente y participativo. (p. 491).

Conforme a lo anterior, los hallazgos que presentamos en este artículo solo son concernientes a la literacidad funcional. Durante el proceso de análisis constantemente nos preguntamos: ¿cuál es el nivel de dominio de la literacidad funcional por parte de los estudiantes de derecho? Como ya expusimos en el apartado metodológico, para el análisis del discurso de los ensayos nos basamos en los tres aspectos propuestos por Castro y Sánchez (2013). Los hallazgos encontrados se presentan organizadamente por secciones siguiendo la propuesta de estos autores y son los siguientes:

\section{Estructura del texto}

No obstante que se les recomendó a los estudiantes redactar su ensayo en lo posible con la estructura de introducción, desarrollo y conclusión, con la finalidad de que pudieran exponer su opinión y reflexionar sobre el tema de una manera más organizada, en la mayoría de los ensayos se identifica que atendieron la estructura. Sin embargo un 
considerable número decidió no hacerlo y siguieron un formato más flexible. 21 trabajos cuentan con la estructura y 15 no la presentan. Esto de ninguna manera afectó el proceso de análisis pues en estos trabajos tratamos de identificar cada parte del texto. En algunos de los ensayos, esto resultó más sencillo.

\section{La introducción de los ensayos}

Respecto al apartado de introducción cabe resaltar que en los discursos en los que los estudiantes sí ponderaron la estructura se observa que en su mayoría plantea el objetivo del ensayo y la relevancia de la argumentación jurídica. Por ejemplo uno de los estudiantes escribió lo siguiente:

En este trabajo hablaré principalmente del impacto que generará el uso de la argumentación jurídica en mi vida profesional y laboral. SI bien, aún no tengo determinado dónde desarrollaré mi vida profesional, ni laboral; me basaré en gran parte en lo aprendido en la clase de argumentación jurídica, [...].

Me veré en el supuesto en el que mi vida profesional y laboral la desarrollaré en cualquier ámbito posible del Derecho [...] y cómo esto me ayudó a entender, sobre todo practicar mi lenguaje corporal, mi seguridad al hablar en público y el cómo formular argumentos convincentes para tratar de captar la atención y confianza del público. (Ensayo del estudiante: Jesús Enrique Torres Nuñez/Arg. Jur/ene-jun de 2020).

En el texto del estudiante se percibe una voz activa con una proyección de vida en el futuro mediano y de largo plazo. Se advierte que, aunque desconoce el espacio laboral posible en el que se desarrollará, tiene claridad en que sus habilidades, destrezas y competencias adquiridas en un curso de Argumentación Jurídica le facilitarán su funcionalidad en el ejercicio profesional. Asimismo, se identifica la forma en que le da importancia a su retórica dialéctica tanto escrita como oral tomando en consideración a su audiencia. Es decir, se identifica en el estudiante un buen dominio de la literacidad funcional indispensable en el campo disciplinar del derecho.

Además, algunos de los estudiantes expusieron los diferentes temas que abordaron y la manera en que organizarían su ensayo. En general, en la mayoría de los trabajos se identificó un buen manejo de la introducción como tal, así como coherencia y claridad en la redacción. También una eficaz comunicación de las ideas. Una de las estudiantes compartió lo siguiente:

El tema por desarrollar consiste en el impacto y la importancia que tiene la argumentación jurídica en mi vida profesional-laboral. Hablaré sobre distintos puntos que conforman a la argumentación, sobre cómo influye en la vida estudiantil y su aplicación en la vida profesional; además se hará uso de los 
apuntes que tomé en la clase de argumentación, lo aprendido en esta materia y sobre cómo me ayudó la realización de los ejercicios prácticos para tener un mejor desempeño en mi vida y sobre todo que esto me auxilió en las demás materias. Es relevante la realización de este ensayo porque voy a poder hacer una retroalimentación sobre todo lo que investigué y aprendí durante el semestre para considerar la argumentación como un factor muy importante para desarrollarse en el área laboral. También plantearé lo investigado y citaré a los autores que estudié en el transcurso de mis clases, que también fueron factores muy importantes para ver la argumentación no solo desde un punto de vista, sino desde varios autores donde pude ampliar un poco más mi conocimiento y juntándolos con lo aprendido en clase; pude adoptar una idea de acuerdo con mis aprendizajes.

La forma en que plantearé este trabajo será utilizando los diferentes factores que conforman a la argumentación jurídica, estos factores son los que conforme avanzaba mi semestre fui aprendiendo en la materia y de las investigaciones y trabajos realizados en clase. Primero que nada, quiero explicar en qué consiste cada factor que aprendí y quiero dar a entender porqué estos factores influyen mucho en la forma en cómo argumentamos y cómo estos nos ayudarán a tener un mejor desempeño en el ejercicio profesional, el impacto que también tiene la argumentación en el desarrollo de nuestra carrera hasta el ejercicio; pero sobre todo en el área de los juicios orales que son los que actualmente en nuestro sistema procesal se aplican (Ensayo de la estudiante: Carolina Ayala Ramírez/Arg. Jur/ene-jun de 2020).

Como vemos, están presentes las categorías discursivas "importancia de la argumentación" y "vida profesional-laboral" en el discurso de la estudiante. Se observa además una escritura organizada, que es indispensable en el ejercicio del derecho. Además se percibe que la estudiante hace una vinculación entre el espacio o contexto escolar y el laboral. Esto es de resaltarse porque en su discurso se identifica que tiene claro cómo las habilidades, destrezas y competencias desarrolladas durante su formación académica inciden en su funcionalidad en el ámbito jurídico-profesional. Además su discurso da cuenta de la relevancia de las prácticas de literacidad académica en las disciplinas. Aunado a lo anterior, se observa en el discurso de la estudiante el papel medular de la argumentación jurídica como actividad lingüística oral en razón de su utilidad para los juicios de este tipo. Una cuestión que incide en la valoración de su dominio de literacidad funcional, específicamente, respecto a la dimensión habilitadora.

Además de los ensayos que fueron referidos utilizando extractos del discurso, cabe señalar que la mayoría de los estudiantes que atendieron la estructura tripartita (introducción, desarrollo y conclusiones) presentaron un discurso consistente y se observó desde los primeros párrafos la inclusión de un lenguaje adecuado para la 
disciplina, el cual fueron adoptando durante el proceso de enseñanza-aprendizaje de la asignatura de Argumentación Jurídica.

En cuanto a los 15 ensayos de los estudiantes que no hicieron uso de la estructura se identificó que, salvo excepciones, la extensa mayoría no refiere un objetivo del ensayo, pero sí plantea al inicio la importancia de la argumentación. Un ejemplo muy claro de esto fue:

La argumentación es sumamente importante en nuestra vida. Se encuentra presente en el día a día; nos encontramos con ella cada que en una plática con otras personas nosotros al pensar distinto tratamos de convencer a los demás de nuestro punto, cuando un comerciante al intentar vendernos algo nos argumenta porqué su producto nos conviene, cuando en comerciales en televisión los candidatos a un puesto político nos tratan de inclinar a dar nuestro voto, cuando nos marcan para sugerir un cambio de compañía telefónica. En todas estas actividades existe una serie de comunes denominadores, como por ejemplo, el intento de imponer una nueva idea, o el propósito de convencer a la otra parte; por lo tanto en este proceso es necesario el comunicar ideas que sean creíbles, coherentes y en su mayor parte posible, ciertas.

En el estudio del derecho, hay una serie completa de ramas distintas como lo son el derecho civil, el derecho mercantil, el derecho penal, derecho constitucional y más, y también existen una serie de disciplinas como lo es la argumentación jurídica. El dominio correcto de la argumentación jurídica resulta ser un pilar básico en el desarrollo de cualquier estudiante de derecho, ya que si este no domina esta disciplina no le servirá de mucho todo lo aprendido en el trascurso por su paso en la formación universitaria, ya que, en la vida laboral no sabrá comunicar, hacer uso y mucho menos transferir de manera eficiente todos sus conocimientos. El tema por desarrollar consiste en el impacto y la importancia que tiene la argumentación jurídica en mi vida profesional-laboral. Hablaré sobre distintos puntos que conforman a la argumentación. Sobre cómo influye en la vida estudiantil y su aplicación en la vida profesional; además se hará uso de los apuntes que tomé en la clase de argumentación, lo aprendido en esta materia y sobre cómo me ayudó la realización de los ejercicios prácticos para tener un mejor desempeño en mi vida y sobre todo que esto me auxilió en las demás materias. (Ensayo de la estudiante: Ana Fernanda Betancourt Aguilera/Arg. Jur/ene-jun de 2020).

En su discurso se observa una clara internalización de la estudiante respecto a los diferentes ámbitos en los que acude a la argumentación no solo en su vida cotidiana, sino en su proceso de formación académica y posteriormente el impacto que tendrá en su vida profesional. Además se percibe cómo se vincula la argumentación jurídica como 
disciplina en otras ramas del derecho para el ejercicio profesional. Su discurso da cuenta del dominio de la literacidad funcional a partir de las habilidades que se pueden adquirir o desarrollar en una materia en particular mediante prácticas de literacidad académica.

La mayoría de trabajos en los que no se utilizó la estructura tripartita tampoco refieren cómo sería organizado el ensayo. Sin embargo, se observa en la mayoría de los ensayos la inclusión de un lenguaje adecuado a la disciplina de la asignatura cursada, así como una redacción clara y coherente en los párrafos del ensayo que pudieran identificarse como introductorios.

\section{El desarrollo de los ensayos}

De igual manera que en el apartado de la introducción, en los ensayos caracterizados por la falta de estructura tripartita tuvimos que encontrar la separación tácita para el análisis del apartado del desarrollo. En esta parte del ensayo, 12 estudiantes perdieron el foco central pues, aunque manifestaron que su trabajo versaría sobre el impacto e importancia de la argumentación jurídica en su vida laboral-profesional, no cumplieron con la promesa del hilo conductor y terminaron por desviarse a temas particulares de la asignatura o a prácticas pedagógicas realizadas durante las sesiones del curso. En este sentido, 24 ensayos del corpus analizado sí fueron consistentes con el tema principal relacionado con alguna de las vertientes como el ejercicio de la abogacía, la función jurisdiccional, la actividad legislativa, etc.

En la redacción de los discursos se observa que unos son expositivos, es decir, narran de manera informativa, descriptiva o enunciativa, mientras que otros son reflexivos porque los estudiantes recurrieron a una discusión más profunda, ya sea en una o varias partes del ensayo, en tanto que en otros de los trabajos se identificó una redacción más argumentativa porque desde nuestra percepción los estudiantes trataron de posicionar alguna idea o aspecto, aunque nunca confrontaron alguna postura de los autores. Un ejemplo de los ensayos más argumentativos es el siguiente:

Estos tres tipos de escenarios son diferentes entre sí, por eso es importante conocer cuál es tipo de auditorio en donde nosotros queremos exponer nuestros argumentos. Es lo que Chaim Perelman mencionaba en sus teorías sobre la argumentación jurídica. Por ejemplo, si estamos en un juicio político, que es el "procedimiento para fincar responsabilidad política u oficial a un servidor público" (Valadés y Gamboa, 2007); ese auditorio será completamente diferente al de una impugnación en el Tribunal Electoral porque son materias divergentes y el asunto que se va a argumentar en el auditorio, también es distinto. Por un lado tenemos a un servidor público, digamos que el juicio es porque se le está acusando de corrupción, entonces tienes que prepararte para que el Congreso te 
escuche y entienda lo que quieres decir, ya sea la parte acusada o el acusador. Además, el Congreso tiene un número de integrantes muchísimo mayor que al de un Tribunal, el Tribunal Electoral no tiene la competencia de darle continuidad a un juicio político, pero sí al de una impugnación de las elecciones de un diputado. Lo que quiero llegar a esto, es que los auditorios universales con mas personas, tiene más variedad de público (Perelman y Olbrechts-Tyteca, 1989), entonces la actividad de argumentar para persuadirlos es más elevada y tiene que ser preparada, pero tiene que preparar conforme a la audiencia que se pretende persuadir. (Ensayo de la estudiante: Itzel Zamira Cumplido Serrano/Arg. Jur/ene-jun de 2020).

El análisis del discurso refleja una apropiación del conocimiento en diferentes áreas del derecho por parte de la estudiante, así como la habilidad y destreza para incorporar la argumentación jurídica en el análisis de aspectos particulares de aquellas. Del discurso se desprende la configuración de argumentos de autoridad, entre otros. Se identifica el uso de teoría de la argumentación para explicar casos reales y prácticos. Conjunta en su discurso los espacios escolares con los profesionales por lo que también muestra cómo el dominio de la literacidad funcional en el contexto de la literacidad disciplinar le será útil para una eficaz funcionalidad en el ámbito social de lo jurídico.

Además, derivado del análisis de los demás ensayos encontramos que el tipo de discurso más utilizado por los estudiantes fue de carácter reflexivo. En menor cantidad se posicionaron en un discurso argumentativo y algunos emitieron su discurso de manera expositiva. Asimismo, en siete ensayos se identificó la combinación de dos tipos de discurso en los que sobresalen el expositivo-reflexivo y el argumentativo-reflexivo.

En el $61 \%$ de la unidad de análisis se presentan referencias teóricas. Pero llama la atención que, aunque se recomendaron durante el curso de Argumentación Jurídica varios autores medulares al respecto, en la mayoría de este porcentaje de ensayos solo se recurrió a algunos cuantos. En el porcentaje restante, se observó que en algunos de los trabajos se mencionan los nombres de los autores; pero no sus ideas, planteamientos o trabajos. Cabe destacar -como se advierte en el apartado metodológico- que los estudiantes tenían la libertad de decidir sustentar o apoyar el contenido del ensayo en al menos uno de los siguientes rubros: a) las lecturas (libros, capítulos de libros, artículos de revistas, etc.) correspondientes a los autores vistos en las sesiones de clase, b) las anotaciones o reflexiones que cada estudiante hizo a partir de la exposición del profesor en las clases y c) las dinámicas pedagógicas consistentes en diferentes ejercicios argumentativos realizados en las clases. Partimos de suponer que redactarían el ensayo con base en las lecturas (de las cuales se les pidió resúmenes en varias ocasiones); sin embargo, del análisis se desprende que la extensa mayoría fundamentalmente hizo referencia al tercer inciso, incluyendo los estudiantes que sí recurrieron a la teoría. Esto nos permite afirmar que los estudiantes prefirieron escribir atendiendo a los ejercicios 
en clase de argumentación jurídica que a lo tratado por la teoría. Esto de cierta forma es preocupante dado que en este artículo referimos que la literatura especializada sostiene que el derecho es argumentación y esta una actividad lingüística; así como también que en la formación académica de los estudiantes de derecho es trascendente la lectoescritura y su desarrollo.

Finalmente, podemos afirmar que los estudiantes presentaron en general una eficaz comunicación de las ideas, con excepción de uno de los trabajos que se caracteriza por ser solo un conglomerado de temas vistos durante el curso. Incluso no encuadra en el género discursivo de ensayo y denota serios problemas de redacción que advierten deficiencias de literacidad.

La conclusión de los ensayos

Al analizar el discurso de este apartado encontramos que en 29 de los trabajos de la unidad de análisis se mencionó la importancia de la argumentación jurídica en la vida laboral-profesional. Esto concuerda con los datos obtenidos al analizar el apartado del desarrollo porque en 24 ensayos los estudiantes se mantuvieron congruentes con el tema principal.

Otro de los hallazgos consistió en que las conclusiones breves fueron la tendencia. Pareciera que los estudiantes se limitaron a finalizar el ensayo y no a profundizar lo suficiente. Mientras algunos estudiantes manejaron un discurso con recomendaciones triviales, otros recapitularon. En tanto que en cuatro trabajos del corpus analizado no se observaron reflexiones conclusivas aunque existiera un apartado especial. El discurso presentado en las conclusiones fue fundamentalmente expositivo. Solo en cerca de una tercera parte de los ensayos los estudiantes mostraron interés para elevar la exposición a matices más reflexivos.

En general, no se observaron aportes sobresalientes en este apartado. En la mayoría de los ensayos que comprenden la unidad de análisis se observó la referencia a que la argumentación jurídica debe estar presente en la formación académica de los estudiantes de derecho para un mejor desempeño profesional. Podemos decir que la mayoría hizo un cierre adecuado, no profundo, pero al menos el apartado encuadra en lo que se considera debe contener una conclusión.

\section{Inserción y manejo de voces}

Castro y Sánchez (2013) precisan que la presencia de referencias teóricas o de argumentos de autoridad en los trabajos académicos de los estudiantes revela la realización de tareas de investigación que fortalecen sus ideas o reflexiones. En los ensayos en los que se encontraron referencias a autores -61\%- se identificó que la mayoría citó las ideas de varios autores para presentar algunos conceptos de argumentación jurídica o para expresar que estaban de acuerdo con sus planteamientos. 
Como esto fue una constante, sobresalieron dos ensayos en los que los estudiantes refirieron múltiples datos teóricos y realizaron un diálogo consistente con los autores. En ninguno de los 36 discursos, aunque algunos eran reflexivos, se confrontaron las posturas de los autores.

En los ensayos que contenían referencias teóricas, la extensa mayoría de los estudiantes utilizó el estilo de la Asociación Americana de Psicología (APA por sus siglas en inglés); sin embargo la generalidad no lo hizo adecuadamente; por ejemplo, al referir una cita textual no señalaron la página donde se encontraba. Solo en uno de los ensayos se observó el manejo de citas de manera sobresaliente. El trabajo corresponde a uno de los estudiantes que sí tuvieron una interacción dialógica eficaz con los autores a los que recurrieron para entretejer su discurso.

Asimismo, independientemente de las problemáticas formales al citar, que desde luego no podemos pasar por inadvertido debido al nivel intermedio en el que se supone se encuentran los estudiantes que cursaron la asignatura, estos en general insertaron correctamente la voz de los otros dentro de su propia voz de manera fluida. Esto representó un manejo regular o adecuado de las habilidades para la lecto-escritura en la unidad de análisis. Como advierten Castro y Sánchez (2013), incorporar las voces ajenas es:

un recurso esencial del discurso académico pues permite la reproducción del pensamiento y conocimiento ajeno, generalmente de un experto. La inclusión de citas no es un mecanismo de fácil manejo; requiere la creación de un contexto adecuado a los efectos de lograr su integración con el nuevo texto. Para tal fin, el escritor debe seleccionar los aspectos que considera relevantes para respaldar una finalidad argumentativa que ha elaborado de antemano. Al extraer un segmento de discurso que ya posee significado, debe realizar los ajustes necesarios y las transformaciones pertinentes para hacerlo funcionar y significar en un nuevo discurso, de modo que las palabras o ideas ajenas se fundan o se integren a las propias en una suerte de diálogo creativo. (p. 496).

Finalmente, se identificó en poco más de la mitad de los ensayos la ausencia de un apartado especial de bibliografía, así como varias deficiencias al respecto. El hallazgo denota habilidades incipientes en trabajos de investigación y la necesidad de fortalecer estas habilidades durante su formación académica.

\section{Construcción de perspectiva}

Como ya fue expuesto, durante el proceso de análisis encontramos que algunos ensayos se redactaron bajo un tipo de discurso reflexivo (en total 13), otros se posicionaron en uno más argumentativo (nueve) y algunos se construyeron de manera expositiva (siete). Además en siete se observó un discurso bajo la dualidad expositivo-reflexivo o ya sea 
reflexivo-argumentativo. En este sentido y conforme a lo que Castro y Sánchez (2013) señalan como construcción de perspectiva, consideramos que estuvo presente de forma más notoria en aquellos discursos redactados con una visión reflexiva completa o parcial. En estos ensayos pudimos encontrar una expresión de opiniones más elaborada y con mayor apropiación del conocimiento de la asignatura de argumentación jurídica adhiriéndose a los autores. De lo anterior se desprende que no obstante se observa cierta construcción de perspectiva en cerca de la mitad de los estudiantes, esta no es autónoma porque está vinculada a argumentos de autoridad.

\section{CONCLUSIONES}

A partir de los resultados encontrados en el corpus de análisis se puede afirmar que en la mayoría de los ensayos redactados como trabajo final para la clase de Argumentación Jurídica descrita en el apartado correspondiente de este artículo, se encontraron hallazgos relacionados al dominio de literacidad funcional por parte de los estudiantes de derecho que los elaboraron. Esto es enriquecedor para el campo de la literacidad en el derecho o la literacidad jurídica porque implicó la presencia de una literacidad funcional en el contexto de las prácticas de literacidad académica en la disciplina durante la formación académica y sobre todo en la asignatura de Argumentación Jurídica. Lo que no solo implica el desarrollo de habilidades, destrezas y capacidades de los estudiantes, sino el hecho de la posibilidad de ponerlas en práctica en la vida profesional-laboral. Por tanto los sitúa como personas capaces de responder a las exigencias de la comunidad en el contexto de su ámbito jurídico profesional.

Además, a partir del estudio se puede concluir que no obstante que la mayoría de la los trabajos que comprenden la unidad de análisis tiene una redacción adecuada, coherencia y claridad en las ideas, así como un manejo de voces ajenas apropiado, es pertinente darle continuidad al desarrollo de habilidades y destrezas de comprensión y producción textual de los estudiantes para un mejor aprendizaje del derecho y su ejercicio profesional, así como el desarrollo de competencias que mejoren el nivel de reflexión y comprensión de textos. Un aspecto de suma relevancia en el área jurídica. Esto porque solo en poco más de la mitad de los ensayos del corpus los estudiantes manifestaron opiniones reflexivas y exclusivamente el $61 \%$ hace referencias teóricas de la argumentación jurídica.

Por tanto, a partir del análisis coincidimos con la literatura publicada en que en el campo del derecho resulta imprescindible fortalecer las competencias comunicativasnarrativas y las destrezas argumentativas de los estudiantes por una razón fundamental: el derecho es lenguaje. Está lleno de proposiciones normativas que al final de cuentas se traducen en proposiciones normativas. La técnica legislativa requiere, entre otras cosas, de sentidos lingüísticos al momento de redactarse la norma jurídica. La interpretación del derecho amerita ejecutar un cierto nivel de reflexión y 
comprensión de textos. La aplicación del derecho implica lo anterior y posteriormente construir un discurso jurídico escrito argumentado en el que se expresan razones. La argumentación jurídica es una actividad lingüística porque se van tejiendo argumentos por medio del lenguaje. Desde luego la comunidad jurídica debe manejarlo de manera conveniente. Sobre todo el que corresponde a la disciplina. Esto solo se consigue fortaleciendo el dominio de la literacidad funcional de los estudiantes de derecho.

Debido a que los hallazgos presentados en este artículo se derivan de una investigación cualitativa, de corte interpretativo, a partir del análisis del discurso de una unidad de análisis, no es posible generalizar las conclusiones; sin embargo pueden ser ponderados en otros programas de derecho de otros entornos institucionales. En este sentido, se debe propiciar el desarrollo de habilidades de lecto-escritura desde el comienzo de la formación académica. Se deben generar espacios en cada asignatura para la comprensión de textos y la interpretación jurídica. Además los profesores deben motivar el desarrollo de destrezas en los estudiantes para la comprensión y producción textual de distintos géneros discursivos jurídicos, para mejorar su desempeño argumentativo porque este incidirá en su campo laboral-jurídico.

En suma, consideramos que el nivel de dominio de literacidad funcional en los estudiantes de derecho de la UACJ que redactaron los discursos sobre argumentación jurídica cuyo conjunto integran el corpus es satisfactorio y apropiado. Sin embargo es conveniente continuar con su desarrollo de una manera más consistente y organizada. Además, debido a lo pertinente que resulta el adecuado manejo de la comprensión y producción textual en los estudiantes de derecho, así como la imperiosa necesidad de elevar su capacidad para reflexionar y comprender discursos jurídicos, podemos afirmar con sustento en el presente estudio -a través del análisis del discurso de 36 ensayos jurídicos- que la literacidad funcional incide favorablemente en un mejor desempeño de los estudiantes en lo que se refiere a su argumentación jurídica.

\section{REFERENCIAS}

Aarnio, A. (2008). Derecho, racionalidad y comunicación social. Ensayos sobre Filosofía del Derecho. Fontamara.

Aguilar, P. (2017). Una propuesta de géneros discursivos escritos del ámbito universitario, jurídico y chileno, orientada a la alfabetización académica de estudiantes de derecho. Perfiles Educativos, 39(155), 179-192. https://doi.org/10.22201/iisue.24486167e.2017.155.58063.

Aguirre, J. (2008). La relación lenguaje y derecho: Jürgen Habermas y el debate iusfilosófico. Opinión Jurídica, $7(13)$,

139-162. https://revistas.udem.edu.co/index.php/opinion/article/view/101.

Atienza, M. (2006). El Derecho como argumentación. Editorial Ariel. 
Atienza, M. (2013). Curso de argumentación jurídica. Trotta.

Baquero, J.M., y Pardo, J.F. (1997). Textos científicos y argumentativos. Una metodología para su comprensión y redacción. Ánfora de la Ciencias Sociales y Humanas, 5(10), 5671. https://doi.org/10.30854/anf.v5.n10.1997.369.

Baquero, J.M., y Pardo, J.F. (1998). El texto jurídico argumentativo: cuestiones de comprensión y redacción. Ediciones Gustavo Ibáñez.

Betancourt, R., y Frías, L. (2015). Competencias argumentativas de los estudiantes de derecho en el marco de las pruebas Saber-Pro. Civilizar, 15(28), 213-228. https://doi.org/10.22518/16578953.289.

Braslavsky, B. (2003). ¿Qué se entiende por alfabetización? Lectura y Vida, 24(2). http://www.lecturayvida.fahce.unlp.edu.ar/numeros/a24n2/24_02_Braslavsky.pdf.

Cáceres, E. (2000). Lenguaje y derecho. Las normas jurídicas como sistema de enunciados. Universidad Nacional Autónoma de México.

Calsamiglia, H., y Tusón, A. (2012) Las cosas del decir. Manual de análisis del discurso (3ª ed.). Editorial Ariel.

Camarillo, H., y Barboza, C. (2020). El aprendizaje disruptivo y expandido del derecho. Profesión tradicional y cultura digital. Sinéctica, (54), 1-16. https://sinectica.iteso.mx/index.php/SINECTICA/article/view/1023/1219.

Carbonell, M. (2014). Cartas a un estudiante de derecho. Editorial Porrúa-Universidad Nacional Autónoma de México.

Cárdenas, J. (2018). La argumentación como derecho. Universidad Nacional Autónoma de México.

Carlino, P. (2013). Alfabetización académica diez años después. Revista Mexicana de Investigación Educativa, 18(57), 355-381. http://www.comie.org.mx/revista/v2018/rmie/index.php/nrmie/article/view/250.

Cassany, D. (2005). Los significados de la comprensión crítica. Lectura y Vida, 26(3), 32-45. http://www.lecturayvida.fahce.unlp.edu.ar/numeros/a26n3/26_03_Cassany.pdf.

Cassany, D. (2006). Tras las líneas. Sobre la lectura contemporánea. Anagrama.

Cassany, D., y Castellà, J. (2010). Aproximación a la literacidad crítica. Perspectiva, 28(2), 353-374. https://doi.org/10.5007/2175-795X.2010v28n2p353.

Castro, M., Hernández, L., y Sánchez, M. (2010). El ensayo como género académico: Una aproximación a las prácticas de escritura de la universidad pública mexicana. En G. Parodi (Ed.), Alfabetización académica y profesional en el siglo XXI: leer y escribir desde las disciplinas (pp. 49-70). Editorial Ariel.

Castro, M., y Sánchez, M. (2013). La expresión de opinión en textos académicos escritos por estudiantes universitarios. Revista Mexicana de Investigación Educativa, 18(57), 483-506.

http://www.comie.org.mx/revista/v2018/rmie/index.php/nrmie/article/view/255. 
Du Bois, J.W. (2003). Discourse and Grammar. En M. Tomasello (Ed.), The New Psychology of Language: Cognitive and Functional Approaches to Language Structure (vol. 2) (pp. 4788). Lawrence Erlbaum.

Du Bois, J.W. (2007). The stance triangle. En R. Englebretson (Ed.), Stancetaking in Discourse Subjectivity, evaluation, interaction (pp. 139-182). https://doi.org/10.1075/pbns.164.07du.

Du Bois, J.W. (2014). Towards a dialogic syntax. Cognitive Linguistics, 25(3), 359-410. https://doi.org/10.1515/cog-2014-0024.

Farfán, E. (2019). La visión integral de la formación jurídica que surge de la retórica. Revista Pedagogía Universitaria y Didáctica del Derecho, 6(2), 5-19. https://doi.org/10.5354/0719-5885.2019.36690.

García Aretio, L. (2019). Necesidad de una educación digital en un mundo digital. Revista $\begin{array}{lllll}\text { Iberoamericana de Educación a Distancia, 22(2), 9-22. } & \end{array}$ http://dx.doi.org/10.5944/ried.22.2.23911.

García Petit, M., y Agüero, C. (2014). Bases para el estudio de la dinámica discursiva en la comunidad jurídica chilena. Revista de Derecho, 27(1), 59-79. https://doi.org/10.4067/S0718-09502014000100003.

Gee, J. (2004). Oralidad y literacidad: de El pensamiento salvaje a Ways with Words. En V. Zavala, M. Niño-Murcia y P. Ames (Eds.), Escritura y sociedad: Nuevas perspectivas teóricas y etnográficas (pp. 23-56). Red para el Desarrollo de las Ciencias Sociales en el Perú.

González de la Torre, Y. (2011). Configuraciones de las prácticas lectoras en contextos sociales. La lectura situada en la escuela y el trabajo. Perfiles Educativos, 33(133). https://doi.org/10.22201/iisue.24486167e.2011.133.27903.

González Lillo, E. (2020). La revisión en escritura colaborativa a través de un wiki: un $\begin{array}{llll}\text { estudio de } \quad \text { caso. } & \text { 4enguaje, }\end{array}$ https://doi.org/10.25100/lenguaje.v48i2.8804.

Guerrero, I. (2019). "No eres tan inteligente, computadora": articulaciones necesarias entre alfabetizaciones digitales y reconfiguración identitaria en la EPJA mexicana. Revista Latinoamericana de Estudios Educativos, 49(1), 131-154. https://rlee.ibero.mx/index.php/rlee/article/view/35.

Henao, J., Londoño-Vásquez, D., y Frías-Cano, L. (2017). Leer y argumentar en un curso de lenguaje para estudiantes de primer año de Derecho de la Institución Universitaria de Envigado (Colombia). Revista Virtual Universidad Católica del Norte, (50),

162-182. http://revistavirtual.ucn.edu.co/index.php/RevistaUCN/article/view/818.

Hernández, G. (2017). Agencia, voz y ethos en conflicto. La escritura académica como experiencia de silenciamiento. En S. Espino y M. Barrón (Coords.), La lectura y la escritura en la educación en México. Aproximaciones teóricas, experiencias aplicadas y perspectivas de futuro (pp. 35-60). IISUE. 
Kalman, J. (1993). En búsqueda de una palabra nueva: la complejidad conceptual y las dimensiones sociales de la alfabetización. Revista Latinoamericana de Estudios Educativos, 23(1), 87-95.

Larraín, A. (2002). Alfabetismo Funcional: Un Desafío de Todos. Psykhe, 11(1), 43-56. http://www.psykhe.cl/index.php/psykhe/article/view/461.

Lea, M.R., y Street, B.V. (2006). The "academic literacies" model: Theory and applications. Theory into Practice, 45(4), 368-377. https://doi.org/10.1207/s15430421tip4504_11.

Londoño-Vásquez, D., Ramírez-Botero, A., y Garay-Herazo, K. (2019). Prácticas de literacidad jurídica en el ejercicio docente en dos facultades de Derecho de Antioquia. Revista Nebrija de Lingüística Aplicada a la Enseñanza de las Lenguas, 13(26), 51-71. Disponible en: https://doi.org/10.26378/rnlael1326313.

Márquez, M., y Valenzuela, J. (2018). Leer más allá de las líneas. Análisis de los procesos de lectura digital desde la perspectiva de la literacidad. Sinéctica, (50), 1-17. https://sinectica.iteso.mx/index.php/SINECTICA/article/view/776.

Montes, M., y López, G. (2017). Literacidad y alfabetización disciplinar: enfoques teóricos y propuestas pedagógicas. Perfiles Educativos, 39(155), 162-178. https://doi.org/10.22201/iisue.24486167e.2017.155.58062.

Moreno, E. (2019). Lectura académica en la formación universitaria: tendencias en investigación. Lenguaje, 47(1), 91-119. https://doi.org/10.25100/lenguaje.v47i1.7180.

Moreno Mosquera, V. (2010). El problema del lenguaje en la interpretación del Derecho: Una reflexión a partir del debate López - Tamayo. Nuevo Derecho, 5(6), 73-85. http://revistas.iue.edu.co/revistasiue/index.php/nuevoderecho/article/view/264.

Moreno Mosquera, V. (2012). De la argumentación pragmadialéctica como competencia investigativa en los estudiantes de derecho de la institución universitaria de envigado. Ratio Juris, 7(15), 123-148. https://doi.org/10.24142/raju.v7n15a6.

Parodi, G. (Ed.). (2010). Alfabetización académica y profesional en el siglo XXI: leer y escribir desde las disciplinas. Editorial Ariel.

Riquelme, A., y Quintero, J. (2017). La literacidad, conceptualizaciones y perspectivas: hacia un estado del arte. Revista Reflexiones, 96(2), 93-105. https://doi.org/10.15517/RR.V96I2.32084.

Salado, L., y Ramírez-Martinell, A. (2018). Capital cultural en el contexto tecnológico: consideraciones para su medición en la educación superior. Revista Iberoamericana $\begin{array}{llll}\text { de Educación } & \text { Superior, } & \text { 125-137. }\end{array}$ https://doi.org/10.22201/iisue.20072872e.2018.24.268.

Salado, L., Ramírez-Martinell, A., y Ochoa, R. (2017). Hábitos de lectura y afinidad tecnológica de los estudiantes universitarios: estudio comparativo de cinco universidades de habla hispana. Estudios Lambda. Teoría y práctica de la didáctica en Lengua y Literatura, (2), 1-24. https://doi.org/10.36799/el.v2i1.43. 
Street, B. (2004). Los Nuevos Estudios de Literacidad. En V. Zavala, M. Niño-Murcia y P. Ames (Eds.), Escritura y sociedad: Nuevas perspectivas teóricas y etnográficas (pp. 81108). Red para el Desarrollo de las Ciencias Sociales en el Perú.

Tapia, E. (2019). Amor, celulares y rezos: prácticas letradas vernáculas en una escuela primaria. Revista Latinoamericana De Estudios Educativos, 49(1), 155-184. https://rlee.ibero.mx/index.php/rlee/article/view/36.

Taranilla, R. (2012). La enseñanza de habilidades comunicativas para la práctica del derecho: la técnica narrativa en contextos judiciales. Revista de Educación y Derecho, (6), 1-25. https://revistes.ub.edu/index.php/RED/article/view/5135.

Tejada, H., y Vargas, A. (2007). Hacia una integración de la literacidad crítica, la literacidad funcional y la literacidad cultural. Lenguaje, 35(2), 197-219. https://doi.org/10.25100/lenguaje.v35i2.4857.

Valerdi, J. (2020). La expresión lingüística de la Actitud en la estructura argumentativa del discurso académico. Lenguaje, 48(1), 01-37. https://doi.org/10.25100/lenguaje.v48i1.8520.

Zavala, V. (2004). Literacidad y desarrollo: los discursos del Programa Nacional de Alfabetización en el Perú. En V. Zavala, M. Niño-Murcia y P. Ames (Eds.), Escritura y sociedad: Nuevas perspectivas teóricas y etnográficas (pp. 437-459). Red para el Desarrollo de las Ciencias Sociales en el Perú. 